\title{
Valve-in-Valve TAVR versus Redo Surgical Aortic Valve Replacement: Early Outcomes
}

\author{
Amila Cizmic ${ }^{1, *}$ Elmar Kuhn ${ }^{1, *}$ Kaveh Eghbalzadeh ${ }^{10}$ Carolyn Weber ${ }^{1}{ }^{10}$ \\ Parwis Baradaran Rahmanian ${ }^{1}$ Matti Adam ${ }^{2}$ Victor Mauri ${ }^{2}$ Tanja Rudolph ${ }^{3}$ Stephan Baldus ${ }^{2}$ \\ Thorsten Wahlers ${ }^{1}$
}

${ }^{1}$ Department of Cardiothoracic Surgery, Heart Center Cologne, Faculty of

Address for correspondence Amila Cizmic, MD, Department of Medicine and University Hospital Cologne, Cologne, Germany

2 Department of Cardiology, Heart Center Cologne, Faculty of

Medicine and University Hospital Cologne, Cologne, Germany

${ }^{3}$ Clinic for General and Interventional Cardiology/Angiology, Heart and Cardiothoracic Surgery, Heart Center Cologne, Faculty of Medicine and University Hospital Cologne, Kerpener Straße 62, 50937 Cologne,

Diabetes Center North Rhine-Westphalia, Bad Oeynhausen, Germany Germany (e-mail: amila.cizmic@icloud.com).

Thorac Cardiovasc Surg 2023;71:94-100.

\begin{abstract}
Objective This study aimed to assess short-term outcomes of patients with failed aortic valve bioprosthesis undergoing valve-in-valve transcatheter aortic valve replacement (ViV-TAVR) or redo surgical aortic valve replacement (rSAVR).

Methods Between 2009 and 2019,90 patients who underwent ViV-TAVR $(n=73)$ or rSAVR ( $n=17$ ) due to failed aortic valve bioprosthesis fulfilled the inclusion criteria. Groups were compared regarding clinical end points, including in-hospital all-cause mortality. Patients with endocarditis and in a need of combined cardiac surgery were excluded from the study. Results ViV-TAVR patients were older $(78.0 \pm 7.4$ vs. $62.1 \pm 16.2$ years, $p=0.012)$ and showed a higher prevalence of baseline comorbidities such as atrial fibrillation, diabetes mellitus, hyperlipidemia, and arterial hypertension. In-hospital all-cause mortality was

Keywords

- valve-in-valve

- redo surgery

- deteriorated aortic valve bioprosthesis

- cardiac surgery

- early outcomes higher for rSAVR than in the ViV-TAVR group (17.6vs. $0 \%, p<0.001$ ), whereas intensive care unit stay was more often complicated by blood transfusions for rSAVR patients without differences in cerebrovascular events. The paravalvular leak was detected in $52.1 \%$ ViVTAVR patients compared with $0 \%$ among rSAVR patients $(p<0.001)$.

Conclusion ViV-TAVR can be a safe and feasible alternative treatment option in patients with degenerated aortic valve bioprosthesis. The choice of treatment should include the patient's individual characteristics considering ViV-TAVR as a standard of care.
\end{abstract}

\section{Introduction}

Redo surgical aortic valve replacement (rSAVR) is associated with incremental operative risk compared with primary SAVR. Data derived from the Society of Thoracic Surgeons

* Both authors contributed equally to the work.

received

December 4, 2020

accepted after revision

May 27, 2021

article published online

September 14, 2021
DOI https://doi.org/ 10.1055/s-0041-1735476. ISSN 0171-6425.
(STS) National Database of patients with previous aortic valve surgery undergoing rSAVR indicates a twofold increase of short-term mortality (4.6\%) compared with first-time SAVR $(2.3 \%) .^{1}$ Valve-in-valve transcatheter aortic valve replacement (ViV-TAVR) for failed bioprosthesis is a less invasive procedure compared with rSAVR, and it is likewise (c) 2021. The Author(s).

This is an open access article published by Thieme under the terms of the Creative Commons Attribution-NonDerivative-NonCommercial-License, permitting copying and reproduction so long as the original work is given appropriate credit. Contents may not be used for commercial purposes, or adapted, remixed, transformed or built upon. (https://creativecommons.org/ licenses/by-nc-nd/4.0/) Georg Thieme Verlag KG, Rüdigerstraße 14, 70469 Stuttgart, Germany 
linked to good short-term outcomes. ${ }^{2}$ Nevertheless, the Valve-in-Valve International Data (VIVID) Registry showed a 7.6\% 30-day mortality in an intermediate to high surgical risk population (STS predicted risk of mortality of $9.8 \%)^{3}$ ViV-TAVR has been associated with procedure-specific complications such as coronary obstruction, high postoperative transvalvular gradients, and cerebrovascular events. ${ }^{4}$

Balancing procedure-specific risks of ViV-TAVR against potential complications associated with rSAVR, ViV-TAVR has increasingly become a valuable alternative to redo surgery. The minimally invasive transcatheter technique offers shorter operation time and less surgical trauma, as well as the avoidance of cardiopulmonary bypass $(\mathrm{CPB}) .^{5}$ Otherwise, redo surgery is still necessary for patients with endocarditis and offers a reasonable treatment option for patients with unfavorable vascular access routes or risks for patient-prosthesis mismatch (PPM). The goal of this study was to compare postoperative outcomes, including in-hospital all-cause mortality of patients with failed aortic valve bioprosthesis undergoing ViV-TAVR or rSAVR at our institution.

\section{Materials and Methods}

All patients enrolled in this retrospective, single-center dataanalysis had previous surgical aortic valve replacement with degeneration of the aortic valve bioprosthesis and the need for reintervention between 2009 and 2019. Stratification to either ViV-TAVR or rSAVR procedure was based on the patient evaluation by the interdisciplinary Heart Team. Patients were identified using the institutional database with the retrospective extraction of relevant data such as preoperative baseline characteristics, procedural data, postoperative complications, and mortality. The main inclusion criteria was a previous surgical aortic valve replacement using a bioprosthesis with the need for reintervention due to stenosis or the insufficiency of the aortic valve bioprosthesis. Patients presenting with bioprosthetic valve endocarditis and in need of combined surgeries were excluded from the study. Finally, 90 patients were selected, of which 73 (81.1\%) patients underwent ViV-TAVR, and 17 (18.9\%) patients underwent rSAVR. The Ethics Committee of the Medical Faculty of the University of Cologne approved this project.

Patients undergoing ViV-TAVR had a preoperative CT angiography to assess annular and aortic root morphology as well as the vascular access route.

In the ViV-TAVR group, the new prosthesis was implanted into the degenerated aortic bioprosthesis using a transfemoral, transaortic, or transapical approach. In the rSAVR group, the degenerated aortic bioprosthesis was replaced through a median sternotomy followed by cardioplegic cardiac arrest and CPB.

\section{Definitions}

All data on perioperative and postinterventional complications were documented and listed according to the Valve Academic Research Consortium-2 consensus (VARC-2). ${ }^{6}$ According to the Acute Kidney Injury Network, acute kidney injury was defined based on RIFLE classification. ${ }^{7,8}$ Heart Team is considered collaboration and dedication across medical specialties to offer optimal patient-centered care, with a requirement that the cardiovascular surgeon and interventional cardiologist jointly participate during the procedure. ${ }^{9}$

\section{Statistics}

Statistical analyses were performed using IBM SPSS Statistics version 26 (IBM Corp, Armonk, New York, United States). Descriptive data for categorical variables of groups were compared using Fisher's exact test, expressed as percentages. Continuous variables indicated with mean \pm standard deviation were compared using the unpaired $t$-test for parametric or the Mann-Whitney U test for non-parametric variables. All reported $p$-values are two-sided, and $p$-values of $<0.05$ were considered statistically significant.

\section{Results}

\section{Baseline Parameters}

Patients included in the study $(n=90)$ were stratified into two groups depending on the selected procedure: ViV-TAVR ( $n=73,81.1 \%)$ and rSAVR group $(n=17,18.9 \%)$. All baseline characteristics are presented in - Table 1. Patients in the ViVTAVR group were older $(78.0 \pm 7.4$ vs. $62.1 \pm 16.2$ years, $p=0.012$ ). ViV-TAVR patients showed a higher prevalence of comorbidities such as atrial fibrillation, diabetes mellitus, hyperlipidemia, and arterial hypertension compared with the rSAVR group. ViV-TAVR patients had significantly more impaired preoperative renal function (53.4 vs. 23.5\%, $p=0.032)$ than the rSAVR patients. The STS risk score showed no significant differences between the two groups $(6.4 \pm 3.1$ vs. $6.4 \pm 3.2, p=0.392$ ). Other clinical baseline characteristics did not differ significantly.

The most common indication for reintervention was stenotic degeneration of the previously implanted bioprosthesis in ViV-TAVR and rSAVR groups.

\section{Surgical and Interventional Parameters}

Group-specific interventional and surgical data are presented in - Table 2. The mean duration of ViV-TAVR procedures was shorter compared with rSAVR operations $(90.8 \pm 35.0$ vs. $220.7 \pm 47.2$ minutes, $p=0.043)$.

In the ViV-TAVR group, the most common vascular access route was femoral (84.9\%), followed by transapical (9.6\%) and transaortic (5.5\%) access. Balloon-expandable valves were implanted in 25 cases (34.2\%) (Edwards Lifesciences, Irvine, California, United States: Sapien 3 and XT), whereas selfexpandable prostheses were implanted in a total of 48 cases (65.8\%) (Medtronic, Minneapolis, United States: CoreValve Evolut; Symetis SA, Ecublens, Switzerland/Boston Scientific, Marlborough, Massachusetts: Acurate neo). The median radiation exposure time was 16 minutes, and contrast media applicated was $101.7 \pm 72 \mathrm{~mL}$. Endotracheal intubation was implemented in 33 patients $(45.2 \%)$ for the ViV-TAVR procedure. ViV-TAVR procedures were complicated by cardiopulmonary resuscitation in four patients (5.5\%). No other 
Table 1 Demographic and preoperative data of patients undergoing ViV-TAVR and rSAVR $(n=90)$

\begin{tabular}{|c|c|c|c|}
\hline & $\begin{array}{l}\text { ViV-TAVR group } \\
(n=73,81.1 \%)\end{array}$ & $\begin{array}{l}\text { rSAVR group } \\
(n=17,18.9 \%)\end{array}$ & p-Value \\
\hline Age (years) & $78.0 \pm 7.4$ & $62.1 \pm 16.2$ & 0.012 \\
\hline Male gender no. (\%) & $32(43.8 \%)$ & $11(64.7 \%)$ & 0.121 \\
\hline $\mathrm{BSA}\left(\mathrm{m}^{2}\right)$ & $1.89 \pm 0.21$ & $1.88 \pm 0.19$ & 0.434 \\
\hline BMI $\left(\mathrm{kg} / \mathrm{m}^{2}\right)$ & $27.0 \pm 5.0$ & $25.9 \pm 5.0$ & 0.434 \\
\hline Coronary artery disease-no. (\%) & $34(46.6 \%)$ & $6(35.3 \%)$ & 0.399 \\
\hline LVEF (\%) & $51.4 \pm 12$ & $51.1 \pm 12$ & 0.224 \\
\hline Previous MI no. (\%) & $17(23.3 \%)$ & $9(52.7 \%)$ & 0.015 \\
\hline Pacer implantation no. (\%) & $9(12.3 \%)$ & $3(17.6 \%)$ & 0.561 \\
\hline Atrial fibrillation no. (\%) & $34(46.6 \%)$ & $2(11.8 \%)$ & 0.008 \\
\hline Diabetes mellitus type II no. (\%) & $31(42.5 \%)$ & $2(11.8 \%)$ & 0.018 \\
\hline Hyperlipidemia no. (\%) & $48(65.8 \%)$ & $5(29.4 \%)$ & 0.006 \\
\hline Arterial hypertension no. (\%) & $70(95.9 \%)$ & $9(52.7 \%)$ & $<0.001$ \\
\hline Pulmonary hypertension no. (\%) & $31(42.5 \%)$ & $3(17.6 \%)$ & 0.057 \\
\hline PAD no. (\%) & $13(17.8 \%)$ & $2(11.8 \%)$ & 0.547 \\
\hline COPD no. (\%) & $20(27.4 \%)$ & $1(5.9 \%)$ & 0.059 \\
\hline Current tobacco use no. (\%) & $7(9.6 \%)$ & $4(23.5 \%)$ & 0.114 \\
\hline TIA no. (\%) & $5(6.8 \%)$ & $0(0 \%)$ & 0.267 \\
\hline Stroke no. (\%) & $9(12.3 \%)$ & $0(0 \%)$ & 0.127 \\
\hline CKD no. (\%) & $39(53.4 \%)$ & $4(23.5 \%)$ & 0.032 \\
\hline Stage I & $12(16.4 \%)$ & $0(0 \%)$ & \\
\hline Stage II & $16(21.2 \%)$ & $3(17.6 \%)$ & \\
\hline Stage III & $8(11 \%)$ & $1(5.9 \%)$ & \\
\hline Stadium IV & $3(4.1 \%)$ & $0(0 \%)$ & \\
\hline STS score & $6.4 \pm 3.1$ & $6.4 \pm 3.2$ & 0.392 \\
\hline
\end{tabular}

Abbreviations: BSA, body surface area; BMI, body mass index; CKD, chronic kidney disease; COPD, chronic obstructive pulmonary disease; LVEF, left ventricular ejection fraction; MI, myocardial infarction; PCI, percutaneous coronary intervention; PAD, peripheral arterial disease; rSAVR, redo surgical aortic valve replacement; TIA, transient ischemic attack; ViV-TAVR, valve-in-valve transcatheter aortic valve replacement.

Table 2 Intraoperative and interventional data of patients undergoing ViV-TAVR and rSAVR $(n=90)$

\begin{tabular}{|c|c|c|c|}
\hline & $\begin{array}{l}\text { ViV-TAVR group } \\
(n=73,81.1 \%)\end{array}$ & $\begin{array}{l}\text { rSAVR group } \\
(n=17,18.9 \%)\end{array}$ & p-Value \\
\hline \multicolumn{4}{|l|}{ Indication for surgery no. (\%) } \\
\hline - Primary AV stenosis & $51(69.9 \%)$ & $11(64.7 \%)$ & 0.679 \\
\hline - Primary AV regurgitation & $22(30.1 \%)$ & $6(35.3 \%)$ & 0.679 \\
\hline Duration (minutes) & $90.8 \pm 35.0$ & $220.7 \pm 47.2$ & 0.043 \\
\hline CPB time (minutes) & - & $118.2 \pm 36.0$ & \\
\hline Cross clamp time (minutes) & - & $71.8 \pm 18.1$ & \\
\hline Access route no. (\%) & & - & \\
\hline - Transfemoral & $62(84.9 \%)$ & - & \\
\hline - Transapical & $7(9.6 \%)$ & - & \\
\hline - Transaortic & $4(5.5 \%)$ & - & \\
\hline
\end{tabular}


Table 2 (Continued)

\begin{tabular}{|c|c|c|c|}
\hline & $\begin{array}{l}\text { ViV-TAVR group } \\
(n=73,81.1 \%)\end{array}$ & $\begin{array}{l}\text { rSAVR group } \\
(n=17,18.9 \%)\end{array}$ & p-Value \\
\hline Implanted prosthesis no. (\%) & & - & \\
\hline - Edwards Sapien XT & $10(13.7 \%)$ & - & \\
\hline - Edwards Sapien 3 & $15(20.5 \%)$ & - & \\
\hline - CoreValve Evolut & $47(64.4 \%)$ & - & \\
\hline - Symetis Accurate Neo & $1(1.4 \%)$ & - & \\
\hline General anesthesia with intubation & $33(45.2 \%)$ & - & \\
\hline Radiation exposure time (minute) & $16[4 ; 53]$ & - & \\
\hline Contrast media (mL) & $101.7 \pm 72$ & - & \\
\hline Cardiopulmonary resuscitation no. (\%) & $4(5.5 \%)$ & - & \\
\hline
\end{tabular}

Abbreviations: AV, aortic valve; CPB, cardiopulmonary bypass; rSAVR, redo surgical aortic valve replacement; ViV-TAVR, valve-in-valve transcatheter aortic valve replacement.

periinterventional complications were observed according to VARC-2 criteria, such as conversion to open-heart surgery, unplanned use of $\mathrm{CPB}$, coronary obstruction, valve malposition, ventricular septal perforation, mitral valve damage, or cardiac tamponade.

In the rSAVR group, the average CPB time was $118.2 \pm 36.0$ minutes, and the average aortic clamp time was $71.8 \pm 18.1$ minutes.

\section{Postoperative Parameters}

Outcome data regarding the postoperative course of patients are displayed in $\mathbf{- T a b l e ~} \mathbf{3}$. The postoperative course of rSAVR patients was complicated by rethoracotomy $(n=2,11.8 \%)$, use of intra-aortic balloon pump (IABP) $(n=1,5.9 \%)$, and extracorporeal membrane oxygenation (ECMO) $(n=2$, $11.8 \%$ ) with no corresponding events in the ViV-TAVR group. Patients in the rSAVR group needed more blood transfusions than the ViV-TAVR patients $(4.1 \pm 8.2$ vs. $0.7 \pm 1.5$ units, $p=0.001)$. Five patients $(6.8 \%)$ in the ViV-TAVR group and one patient $(5.9 \%)$ in the rSAVR group developed a new $3^{\text {rd }}$ degree atrioventricular (AV) block with subsequent implantation of a permanent pacemaker $(p=0.886)$.

Twelve ViV-TAVR patients (16.4\%) developed vascular complications postinverventionally according to VARC-2 criteria. Among them, nine patients (12.3\%) developed an inguinal hematoma at the vascular access site. A pseudoaneurysm of the femoral access artery was observed in three patients (4.1\%). Five patients (6.9\%) underwent a local surgical revision due to persistent bleeding from the transfemoral vascular access site. The incidence of gastrointestinal bleeding showed no significant difference between ViV-TAVR and rSAVR group (1.4 vs. $5.9 \%, p=0.256$ ).

The rate of acute kidney injury (AKI) was similarly distributed among both groups ( 34.7 vs. $29.4 \%, p=0.677$ ), with no significant difference in the rate of new dialysis (5.5 vs. $5.9 \%, p=0.948)$. Patients in the rSAVR group had a longer in-hospital stay than ViV-TAVR patients (12.3 \pm 7.3 vs. $10.6 \pm 9.2$ days, $p=0.316$ ). In-hospital all-cause mortality was significantly lower for ViV-TAVR patients than in rSAVR patients ( 0 vs. $17.6 \%, p<0.001$ ). Two patients died due to fulminant cardiogenic shock, and one patient died due to multiorgan failure because of septic shock.

\section{Echocardiographic Characteristics}

The preoperative left ventricular ejection fraction did not differ significantly between the two groups. Postinterventionally, a mild paravalvular leak (PVL) was present in 32 patients (47.8\%) and a moderate PVL in six patients (9\%) in the ViV-TAVR group, whereas no PVL occurred in the rSAVR group $(p<0.001)$. The mean and maximal transvalvular gradients, as well as the maximal velocity, did not show a significant difference between the two groups; however, the effective orifice area was larger in rSAVR patients compared with ViV-TAVR patients $(1.8 \pm 0.7$ vs. $\left.1.28 \pm 0.4 \mathrm{~cm}^{2}, p=0.716\right)$.

\section{Discussion}

The presented dataset's scope was to analyze short-term outcomes in patients treated for bioprosthetic aortic valve degeneration. Patients with endocarditis or failed mechanical prostheses were excluded from the analysis. All 90 included patients received either ViV-TAVR or redo-surgery, respectively. Both groups include patients with high procedure-specific risks for adverse events and incorporate characteristic differences since ViV-TAVR patients were older than rSAVR patients. The presented results indicate higher in-hospital mortality for rSAVR patients. As rSAVR patients more often had relevant postoperative complications, including higher short-term mortality, ViV-TAVR was linked to the presence of a postinterventional paravalvular leak and slightly higher transvalvular gradients.

The need for strict anticoagulation therapy after mechanical aortic valve replacement combined with the possibility of a ViV-TAVR procedure favors bioprostheses' implantation even in middle-aged patients. Thus, implantation rates of aortic valve bioprostheses currently increase, and consequently, evaluation of the different treatment options for patients with failed aortic valve bioprostheses gains an important role. ${ }^{10}$ As long as randomized controlled trials 
Table 3 Postoperative data of patients undergoing ViV-TAVR and rSAVR $(n=90)$

\begin{tabular}{|c|c|c|c|}
\hline & $\begin{array}{l}\text { ViV-TAVR group } \\
(n=73,81.1 \%)\end{array}$ & $\begin{array}{l}\text { rSAVR group } \\
(n=17,18.9 \%)\end{array}$ & p-Value \\
\hline Postoperative resuscitation no. (\%) & $2(2.7 \%)$ & $0(0 \%)$ & 0.490 \\
\hline Rethoracotomy no. (\%) & - & $2(11.8 \%)$ & - \\
\hline IABP no. (\%) & $0(0 \%)$ & $1(5.9 \%)$ & 0.037 \\
\hline ECMO no. (\%) & $0(0 \%)$ & $2(11.8 \%)$ & 0.003 \\
\hline Blood transfusion (units) & $0.7 \pm 1.5$ & $4.1 \pm 8.2$ & 0.001 \\
\hline New $3^{\text {rd }}$ degree AV block & $5(6.9 \%)$ & $1(5.9 \%)$ & 0.886 \\
\hline New pacemaker implantation no. (\%) & $5(6.9 \%)$ & $1(5.9 \%)$ & 0.886 \\
\hline Tracheostomy no. (\%) & $0(0 \%)$ & $0(0 \%)$ & - \\
\hline Cerebrovascular events no. (\%) & $2(2.7 \%)$ & $0(0 \%)$ & 0.788 \\
\hline - TIA & $1(1.4 \%)$ & $0(0 \%)$ & 0.627 \\
\hline - Stroke & $1(1.4 \%)$ & $0(0 \%)$ & 0.627 \\
\hline Postoperative delirium no. (\%) & $17(23.3 \%)$ & $3(17.6 \%)$ & 0.614 \\
\hline Gl bleeding no. (\%) & $1(1.4 \%)$ & $1(5.9)$ & 0.256 \\
\hline \multicolumn{4}{|l|}{ Vascular complications no. (\%) } \\
\hline - Inguinal hematoma & $9(12.3 \%)$ & - & \\
\hline - Pseudoaneurysm & $3(4.1 \%)$ & - & \\
\hline - Surgical revision of access site & $5(6.9 \%)$ & - & \\
\hline - Intravascular stenting & $1(1.4 \%)$ & - & \\
\hline Acute kidney injury no. (\%) & $25(34.7 \%)$ & $5(29.4 \%)$ & 0.677 \\
\hline - Stage I & $5(6.9 \%)$ & $2(11.8 \%)$ & 0.496 \\
\hline - Stage II & $13(17.8 \%)$ & $1(5.9 \%)$ & 0.222 \\
\hline - Stage III & 7 (9.5\%) & $2(11.8 \%)$ & 0.788 \\
\hline New dialysis no. (\%) & $4(5.5 \%)$ & $1(5.9 \%)$ & 0.948 \\
\hline In-hospital stay (day) & $10.6 \pm 9.2$ & $11.6 \pm 7.3$ & 0.001 \\
\hline ICU stay (day) & $3.0 \pm 4.0$ & $3.4 \pm 3.6$ & 0.126 \\
\hline In-hospital mortality no. (\%) & $0(0 \%)$ & $3(17.6 \%)$ & $<0.001$ \\
\hline Immediate mortality no. (\%) & $0(0 \%)$ & $1(5.9 \%)$ & 0.037 \\
\hline \multicolumn{4}{|l|}{ Postoperative echocardiography } \\
\hline Ejection fraction (\%) & $51.9 \pm 11.9$ & $51.6 \pm 13.0$ & 0.870 \\
\hline Mean gradient $(\mathrm{mm} \mathrm{Hg})$ & $17.2 \pm 10.1$ & $11.0 \pm 6.6$ & 0.503 \\
\hline Max gradient $(\mathrm{mm} \mathrm{Hg})$ & $30.2 \pm 17.4$ & $19.7 \pm 11.2$ & 0.140 \\
\hline Max velocity $(\mathrm{m} / \mathrm{s})$ & $2.7 \pm 0.8$ & $2.1 \pm 0.6$ & 0.828 \\
\hline Effective orifice area $\left(\mathrm{cm}^{2}\right)$ & $1.28 \pm 0.4$ & $1.8 \pm 0.7$ & 0.716 \\
\hline Paravalvular leak no. (\%) & $38(52.1 \%)$ & $0(0 \%)$ & $<0.001$ \\
\hline - Mild & $32(43.8 \%)$ & $0(0 \%)$ & 0.001 \\
\hline - Moderate & $6(8.2 \%)$ & $0(0 \%)$ & 0.221 \\
\hline Moderate PPM & $6(8.2)$ & $5(29.4)$ & 0.016 \\
\hline
\end{tabular}

Abbreviations: AV block, atrioventricular block; ECMO, extracorporeal membrane oxygenation; GI bleeding, gastrointestinal bleeding; IABP, intraaortic balloon pump; Immediate mortality, mortality within 48 hours after the procedure; PPM, patient-prosthesis mismatch; rSAVR, redo surgical aortic valve replacement; TIA, transient ischemic attack; ViV-TAVR, valve-in-valve transcatheter aortic valve replacement.

do not address this issue, retrospective analyses have to guide decision-making.

In an overall comparable patient cohort, Erlebach et al. investigated short-term outcomes and 1-year survival in patients treated for failed surgical bioprosthetic valves by minimally invasive transcatheter technique or redo surgery. Analyzing 102 consecutive patients showed no relevant difference in 30-day all-cause mortality but a significantly 
higher survival rate for redo surgery patients after 1 year. Incidence rates of myocardial infarction and stroke were equally distributed; however, post-procedural need for dialysis and the paravalvular leak was higher in ViV-TAVR patients. As the authors conclude that redo surgery should continue to stay the standard treatment option, they also state that the valve-in-valve technique may be reasonable for a specific subgroup of patients. ${ }^{5}$ In the presented dataset, the mortality rate was in favor of valve-in-valve patients compared with three deaths in the redo surgery group. Thus, our results do not support the assumption of redo-surgery as a standard treatment option. The mortality rate (17.6\%) for rSAVR patients in the presented dataset was higher than previously reported in the literature (2.6-5.7\%). ${ }^{5,11,12}$ This could be attributed to preoperative comorbidities (CKD, LVEF $<35 \%$ ) and postoperative complications that these patients developed (AKI, rethoracotomy, ECMO, and IABP implantation). The higher mortality rate could also be due to the small group size of 17 patients who underwent the rSAVR.

In a larger cohort of 350 patients undergoing ViV-TAVR or redo-surgery due to failed stented aortic bioprosthesis, Sedeek et al. described similar operative mortality in both groups. In contrast, procedure-related complications occurred less frequently in the ViV-TAVR group. ${ }^{12}$ However, the echocardiographic assessment revealed higher transvalvular gradients with higher rates of a severe PPM after ViV-TAVR compared with rSAVR, while aortic regurgitation was remarkably rare $(<1 \%)$ and was equally distributed between the two groups. We similarly detected favorable echocardiographic findings in patients who underwent redo-surgery with lower mean and maximal transvalvular gradients and no paravalvular leak than the ViV-TAVR cohort. However, we could not foresee the long-term effect of marginally higher gradients and an increased rate of paravalvular leaks in the ViV-TAVR group, given the higher shortterm mortality in the operated cohort. ${ }^{13}$

Interestingly, the calculated PPM favored ViV-TAVR patients with six patients with a moderate PPM (8.2 vs. $29.4 \%, p=0.016$ ), which does not explain the postoperative echocardiographic parameters' hemodynamics. Moreover, Bleiziffer et al. showed that neither severe PPM nor elevated gradients were linked to adverse clinical events, including 1year mortality in patients after ViV-TAVR. ${ }^{14}$ These retrospective studies, including the presented dataset, do not include procedures implementing valve fracturing (cracking) to achieve more favorable postinterventional hemodynamic results. ${ }^{15}$ This technique could reduce transvalvular gradients after ViV-TAVR and abolish the advantageous echocardiographic findings for rSAVR in future studies.

The five rSAVR patients (29.4\%) who developed a moderate PPM postoperatively had a smaller new bioprosthesis than the old bioprosthesis. Fallon et al. reported that severe and moderate PPMs increase the mortality risk after a surgical aortic valve replacement. ${ }^{16}$ Moderate PPM could be one of the reasons for the higher mortality in the reported rSAVR group.

Evaluating evidence from retrospective trials covering a total of 500 patients, Tam et al. reported favorable outcomes for ViV-TAVR patients compared with redo surgery patients with degenerated aortic bioprosthesis in a systematic review with meta-analysis. ${ }^{11}$ The authors describe comparable mortality rates but, finally, a lower need for pacemaker implantation and dialysis after the ViV-TAVR. Most recently, a U.S.-nationwide study of matched high-risk patients detected superior outcomes for valve-in-valve technique compared with redo surgery regarding 30-day mortality and bleeding complications. ${ }^{17}$

Several reasons limit the interpretation of our results. The study is a retrospective data analysis simultaneously incorporating imbalances regarding preoperative baseline characteristics of both treatment groups. The patients undergoing ViV-TAVR were markedly older and presented with a higher prevalence of relevant comorbidities. Additionally, none of the ViV-TAVR patients underwent valve fracturing, ultimately resulting in higher postinterventional transvalvular gradients.

In conclusion, our results provide insights into current clinical outcomes for ViV-TAVR and rSAVR patients with higher mortality rates in the redo-group in a single-center setting. Patients presenting with degenerated aortic valve bioprostheses should be treated according to their individual risk profile. ViV-TAVR should become the standard of care for patients with isolated degenerated aortic valve bioprosthesis. rSAVR should be considered an option for patients with unfavorable vascular access or other contraindications for ViV-TAVR.

\section{Authors' Contribution}

Since our author list overcomes the limit of seven authors per manuscript, we would like to elaborate on the individual authors' contribution. A.C. and E.K. have contributed equally to the writing of the manuscript. K.E., C.W., and V.M. did the literature search as well as data extraction. P.B.R. and M.A. made the statistical interpretation of data. T.R., S.B., and T.W. were responsible for the critical revision of the manuscript.

\section{Conflict of Interest}

None declared.

\section{References}

1 Kaneko T, Vassileva CM, Englum B, et al. Contemporary outcomes of repeat aortic valve replacement: a benchmark for transcatheter valve-in-valve procedures. Ann Thorac Surg 2015;100(04): 1298-1304, discussion 1304

2 Silaschi M, Wendler O, Seiffert M, et al. Transcatheter valve-invalve implantation versus redo surgical aortic valve replacement in patients with failed aortic bioprostheses. Interact Cardiovasc Thorac Surg 2017;24(01):63-70

3 Dvir D, Webb J, Brecker S, et al. Transcatheter aortic valve replacement for degenerative bioprosthetic surgical valves: results from the global valve-in-valve registry. Circulation 2012;126(19):2335-2344

4 Dvir D, Leipsic J, Blanke P, et al. Coronary obstruction in transcatheter aortic valve-in-valve implantation: preprocedural evaluation, device selection, protection, and treatment. Circ Cardiovasc Interv 2015;8(01):e002079 
5 Erlebach M, Wottke M, Deutsch MA, et al. Redo aortic valve surgery versus transcatheter valve-in-valve implantation for failing surgical bioprosthetic valves: consecutive patients in a single-center setting. J Thorac Dis 2015;7(09):1494-1500

6 Kappetein AP, Head SJ, Généreux P, et al; Valve Academic Research Consortium (VARC)-2. Updated standardized endpoint definitions for transcatheter aortic valve implantation: the Valve Academic Research Consortium-2 consensus document(VARC-2). Eur J Cardiothorac Surg 2012;42(05):S45-S60

7 Mehta RL, Kellum JA, Shah SV, et al; Acute Kidney Injury Network. Acute kidney injury network: report of an initiative to improve outcomes in acute kidney injury. Crit Care 2007;11(02):R31

8 Lopez-Delgado JC, Esteve F, Torrado $\mathrm{H}$, et al. Influence of acute kidney injury on short- and long-term outcomes in patients undergoing cardiac surgery: risk factors and prognostic value of a modified RIFLE classification. Crit Care 2013;17(06):R293

9 Coylewright M, Mack MJ, Holmes DR Jr, O'Gara PT. A call for an evidence-based approach to the Heart Team for patients with severe aortic stenosis. J Am Coll Cardiol 2015;65(14):1472-1480

10 Bartus K, Litwinowicz R, Bilewska A, et al. Intermediate-term outcomes after aortic valve replacement with a novel RESILIA ${ }^{\mathrm{TM}}$ tissue bioprosthesis. J Thorac Dis 2019;11(07):3039-3046

11 Tam DY, Vo TX, Wijeysundera HC, Dvir D, Friedrich JO, Fremes SE. Transcatheter valve-in-valve versus redo surgical aortic valve replacement for the treatment of degenerated bioprosthetic aortic valve: a systematic review and meta-analysis. Catheter Cardiovasc Interv 2018;92(07):1404-1411

12 Sedeek AF, Greason KL, Sandhu GS, Dearani JA, Holmes DR Jr, Schaff HV. Transcatheter Valve-in-valve vs surgical replacement of failing stented aortic biological valves. Ann Thorac Surg 2019; 108(02):424-430

13 Abdel-Wahab M, Mehilli J, Frerker C, et al; CHOICE investigators. Comparison of balloon-expandable vs self-expandable valves in patients undergoing transcatheter aortic valve replacement: the CHOICE randomized clinical trial. JAMA 2014;311(15): 1503-1514

14 Bleiziffer S, Erlebach M, Simonato M, et al. Incidence, predictors and clinical outcomes of residual stenosis after aortic valve-invalve. Heart 2018;104(10):828-834

15 Saxon JT, Allen KB, Cohen DJ, Chhatriwalla AK. Bioprosthetic valve fracture during valve-in-valve TAVR: bench to bedside. Interv Cardiol (Lond) 2018;13(01):20-26

16 Fallon JM, DeSimone JP, Brennan JM, et al. The incidence and consequence of prosthesis-patient mismatch after surgical aortic valve replacement. Ann Thorac Surg 2018;106(01):14-22

17 Hirji SA, Percy ED, Zogg CK, et al. Comparison of in-hospital outcomes and readmissions for valve-in-valve transcatheter aortic valve replacement vs. reoperative surgical aortic valve replacement: a contemporary assessment of real-world outcomes. Eur Heart J 2020;41(29):2747-2755 